\title{
RAZÃO ENTRE LINFONODOS POSITIVOS E DISSECADOS COMO FATOR PROGNÓSTICO EM CÂNCER DE CÓLON
}

\author{
Damila Cristina trufelli*, Vanessa da Costa Miranda, Cláudia Cristina Palos, Eliete Ramos, Milene Nibi Abräo, Vanessa Assis da \\ Silva, Rachel Riechelmann, Rafael Kaliks, Auro Del Giglio
}

Trabalho realizado na Disciplina de Hematologia e Oncologia da Faculdade de Medicina do ABC e pelo Programa Integrado de Oncologia do Hospital Israelita Albert Einstein, São Paulo, SP

\section{*Correspondência}

Faculdade de Medicina do ABC

Av. Lauro Gomes, 2000

Vila Sacadura Cabral

Santo André - SP

Cep 09060-870

\section{RESUMO}

Oвjetivo. Examinar o impacto da razão entre linfonodos comprometidos e o total de linfonodos dissecados como fator de prognóstico em pacientes com câncer de cólon.

Métodos. Trata-se de um trabalho retrospectivo, realizado por meio da consulta dos prontuários dos pacientes com câncer de cólon, consecutivos, provenientes de três instituições localizadas no ABC paulista. De cada prontuário, foram extraídos dados demográficos e dados relacionados à doença. Estatística descritiva e regressão proporcional de Cox foram utilizadas para descrever os resultados.

Resultados. Foram analisados 106 pacientes no total, com média de idade de 62,82 $\pm 11,6$ anos, sendo a maioria do sexo masculino (53,8\%). A mediana de linfonodos dissecados por paciente foi de II,5 (3 - 45 linfonodos) e 58,5\% tiveram mais que 10 linfonodos dissecados. A média do tempo de seguimento foi de 25,05 \pm ।5,21 meses (2 - 64 meses), sendo que $32,1 \%$ dos pacientes faleceram em consequêencia do tumor. A análise univariada mostrou que a sobrevida dos pacientes incluídos no estudo está relacionada ao quociente linfonodos positivos / linfonodos dissecados $(p=0,044)$, estadiamento da doença $(p=0,00 I)$ e recidiva do tumor $(p=0,058)$. No entanto, na realizaçãa da análise multivariada, observou-se que o único fator independente relacionado à sobrevida para câncer de cólon na população estudada é 0 estadiamento $(p=0,001)$.

Conclusä́o. Neste estudo, o quociente linfonodal não foi associado à sobrevida global de forma independente quando considerado juntamente ao estadiamento em pacientes com carcinoma de cólon. Estudos com número maior de pacientes e prospectivos são necessários para avaliar o real impacto do quociente linfonodal no prognóstico de pacientes com câncer de cólon.

Unitermos: Neoplasia de cólon. Linfonodos. Prognóstico.

\section{INTRODUÇÃO}

O câncer de cólon (CC) é o segundo câncer mais prevalente no mundo depois do câncer de mama'. O CC é o quarto tipo de câncer mais freqüente em homens (antecedido por câncer pulmão, estômago e próstata) e o terceiro tipo mais comum em mulheres (antecedido por câncer de mama e colo de útero) no mundo'. As estimativas de incidência de CC no Brasil para 2006, publicadas pelo Instituo Nacional do Câncer (INCA), apontam que o CC foi - quinto tumor maligno mais freqüente entre homens (com I I.390 casos novos) e o quarto entre as mulheres (I3.970 casos novos)2. Além disso, o CC constitui a terceira maior causa de mortalidade por câncer no Brasil, havendo diferenças entre os sexos: nas mulheres, precedidos por carcinoma de mama e pulmão e, nos homens, por carcinoma de próstata e pulmão². Vários fatores prognósticos parecem influenciar a sobrevida do paciente com CC. A presença de envolvimento linfonodal é indubitavelmente o fator prognóstico mais importante e um dos mais bem estudados ${ }^{3,4}$. Dukes, em 1932, reconheceu pioneiramente a importância do status linfonodal e incorporou-o ao seu sistema de estadiamento para câncer retal| ${ }^{5}$. Em 1939, Mayo e Sympson ${ }^{6}$ aplicaram o sistema de Dukes para estadiamento de câncer de cólon, e todos os outros sistemas subseqüentes, incluindo o atual sistema TNM (tumor- linfonodo-metástase $)^{7}$, continuaram a reconhecer o impacto do envolvimento dos linfonodos na sobrevida do paciente ${ }^{4}$. Desta forma, a presença de metástases linfonodais em pacientes com CC tem implicações prognósticas relevantes quanto à sobrevida global e sobrevida livre de doença $a^{8-10}$.

Apesar da importância da avaliação patológica dos linfonodos (LN) no estadiamento do CC, o número exato de LN que precisa ser ressecado para se determinar corretamente o status linfonodal ainda é controverso. Estudos de pacientes com CC com LN negativos têm reportado que tanto a sobrevida livre de doença quanto a sobrevida global estão associadas a um maior número de $L N$ ressecados $4,11-13$. Alguns pesquisadores advogam que a ampla dissecção linfonodal tem benefício terapêutico, enquanto outros acreditam que tal procedimento provê apenas um estadiamento mais acurado ${ }^{14,15}$. Vale ressaltar que o número de $L N$ ressecados depende da extensão da cirurgia e da habilidade do cirurgião, enquanto que a precisão na avaliação de metástases linfonodais irá depender diretamente da técnica utilizada pelo patologista e de sua experiência ${ }^{4,16}$. Além do número total de $L N$ ressecados, a relação entre o número de $L N$ acometidos pela neoplasia e o número de $\mathrm{LN}$ ressecados (quociente linfonodal) também poderia fornecer informações prognosticas importantes para pacientes com CC. Em 


\begin{tabular}{|c|c|}
\hline Característica & $\mathbf{N}(\%)$ \\
\hline Idade* & $62,82 \pm 11,6$ \\
\hline \multicolumn{2}{|l|}{ Sexo } \\
\hline Masculino & $57(53,8 \%)$ \\
\hline Feminino & $49(46,2 \%)$ \\
\hline \multicolumn{2}{|l|}{ Raça } \\
\hline Branca & $50(83,3 \%)$ \\
\hline Não-branca & $10(16,7 \%)$ \\
\hline \multicolumn{2}{|c|}{ História familiar de qualquer câncer } \\
\hline Positiva & $27(69,2 \%)$ \\
\hline Negativa & $12(30,8 \%)$ \\
\hline \multicolumn{2}{|c|}{ Localização anatômica do tumor primário } \\
\hline Cólon direito & $34(34 \%)$ \\
\hline Cólon esquerdo & $20(20 \%)$ \\
\hline Cólontransverso & $13(13 \%)$ \\
\hline Sigmóide & $33(33 \%)$ \\
\hline \multicolumn{2}{|l|}{ Diferenciação } \\
\hline Pouco diferenciado & $5(5,2 \%)$ \\
\hline Moderadamente diferenciado & $86(88,7 \%)$ \\
\hline Bem diferenciado & $6(6,2 \%)$ \\
\hline \multicolumn{2}{|l|}{ Estadiamento } \\
\hline Estadio I & $2(1,9 \%)$ \\
\hline Estadio II & $24(22,9 \%)$ \\
\hline Estadio III & $18(17,1 \%)$ \\
\hline Estadio IV & $61(58,1 \%)$ \\
\hline Linfonodos positivos $*$ ** & $\mid(0-14)$ \\
\hline Linfonodos dissecados** & $11,5(3-45)$ \\
\hline QuocienteP/D* & $0,19 \pm 0,25$ \\
\hline
\end{tabular}

LEGENDA: quociente $P / D$ = quociente entre linfonodos positivos edissecados

*Média \pm desvio padrão; ***Mediana(mín-máx)/NOTA: Ototal de pacientesavaliados para cadavariável éinferiora 106, pois para algumas destas características não haviainformações no prontuário.

um estudo conduzido pelo nosso grupo com pacientes diagnosticadas com câncer de mama, foi possível estabelecer uma relação significativa entre a razão de linfonodos acometidos/ linfonodos dissecados e a sobrevida livre de doença ${ }^{17}$. Para pacientes com câncer colorretal, alguns estudos mostraram também uma associação positiva entre a sobrevida global e o quociente

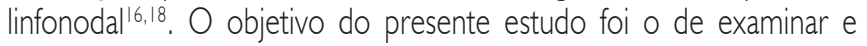
confirmar o impacto da razão entre LN positivos e o total de LN ressecados no prognóstico dos pacientes com câncer de cólon em nosso meio, principalmente no que diz respeito à sobrevida global.

\section{Métodos}

Trata-se de um trabalho retrospectivo, aprovado pelo Comitê de Ética e Pesquisa da Faculdade de Medicina do $A B C$, realizado por meio da consulta dos prontuários dos pacientes com câncer de cólon consecutivos, provenientes do Instituto de Oncologia da Faculdade de Medicina do ABC, do Hospital de Ensino Padre Anchieta e do Hospital Estadual Mário Covas, atendidos de março de 2001 a agosto de 2006. Os pacientes foram identificados por registros do serviço de oncologia de cada hospital incluído no estudo. Pacientes com mais de 18 anos e com diagnóstico de tumor de cólon de qualquer estadio foram considerados elegíveis. Foram excluídos os pacientes com prontuários que não continham dados acerca do número de linfonodos dissecados (anatomopatológico não existente) e aqueles em que não houve dissecção de linfonodos. Excluíram-se ainda casos que não foram operados com a finalidade de ressecção do tumor primário.

De cada prontuário, foram obtidos os seguintes dados: idade, sexo, história familiar de neoplasia maligna, grau de diferenciação do tumor, estadiamento patológico TNM, localização anatômica do tumor, número de linfonodos acometidos e de linfonodos ressecados, tempo de seguimento após a cirurgia do CC (em meses), se houve óbito e data do óbito, presença de metástase à distância no diagnóstico e presença de recidiva loco-regional. A sobrevida livre de doença não foi calculada devido à falta de informações nos prontuários analisados acerca da data da recidiva da doença.

Estatística descritiva foi utilizada para descrever as características da casuística e os dados relacionados ao tumor primário. A sobrevida global foi estimada através do método de Kaplan-Meier. A sobrevida foi definida da seguinte forma: data da última consulta (censurados) ou do óbito menos a data da cirurgia para ressecção do tumor primário. O quociente linfonodal foi considerado como uma variável contínua. As análises estatísticas univariada e multivariada foram realizadas para todas as variáveis pelo teste Cox proportional hazard regression, na tentativa de predizer os fatores de risco independentes relacionados à sobrevida global da população estudada. Adotou-se o valor de significância estatística menor ou igual a 10\% (pd"0,I) para a análise univariada, e menor ou igual a 5\% (pd"0,05) para a análise multivariada. Os testes estatísticos foram realizados a partir do programa SPSS ${ }^{\circledR}$ versão 13.0 (SPSS ${ }^{\circledR}$ Inc; llinois, USA).

\section{Resultados}

Foram incluídos para análise 106 pacientes. A média de idade foi $62,82 \pm$ 11,6 anos e a maioria dos pacientes era do sexo masculino (53,8\%), da raça branca $(83,3 \%)$ e com história familiar positiva para qualquer câncer $(69,2 \%)$. O tumor se localizava predominantemente no cólon direito (34\%) e sigmóide (33\%) e a maioria era moderadamente diferenciado $(88,7 \%)$. A mediana de linfonodos dissecados e acometidos foi, respectivamente, de II,5 (mínimo de 3 e máximo de 45) e um (mínimo de 0 e máximo de 14), sendo que mais da metade dos pacientes se encontrava no estadio IV da doença (58,1\%). A média do tempo de seguimento foi de 25,05 \pm 15,21 meses ( 2 - 64 meses). A sobrevida global média foi de 42,8 $\pm 2,43$ meses. A Tabela I descreve as características clínicas e demográficas dos pacientes incluídos no estudo com mais detalhes.

A análise univariada mostrou que a sobrevida global dos pacientes incluídos no estudo está associada ao quociente de linfonodos positivos/linfonodos dissecados, ao estadiamento da doença e à recidiva do tumor. No entanto, na análise multivariada, observouse que o único fator independente relacionado à sobrevida para câncer de cólon na população estudada foi o estadiamento $(p=0,00 \mathrm{l})$. Ao analisar apenas os pacientes com no mínimo 10 


\begin{tabular}{|c|c|c|c|c|}
\hline \multicolumn{5}{|c|}{$\begin{array}{l}\text { Tabela 2-Valores de significância estatística para as análises } \\
\text { univariada e multivariada }\end{array}$} \\
\hline \multirow[t]{2}{*}{ Variável } & \multicolumn{2}{|c|}{ Univariada } & \multicolumn{2}{|c|}{ Multivariada } \\
\hline & $\mathrm{p}^{*}$ & $\operatorname{Exp}(B)$ & $\mathrm{p}^{*}$ & $\operatorname{Exp}(B)$ \\
\hline QuocienteP/D & 0,044 & 4,154 & 0,205 & 2,620 \\
\hline Estadiamento & 0,001 & 4,922 & 0,001 & 4,900 \\
\hline Recidiva & 0,058 & 0,491 & 0,267 & 0,643 \\
\hline
\end{tabular}

*Coxproportional hazard regression/

LEGENDA: quocienteP/D = quociente entre o número de linfonodos acometidos e dissecados

linfonodos dissecados (62 pacientes), observou-se que os resultados se mantiveram semelhantes aos descritos anteriormente, e não serão apresentados. Contudo, a pequena amostra de pacientes limita qualquer interpretação definitiva. Os valores de significância estatística estão descritos na Tabela 2.

\section{Discussão}

Segundo Sigurdson et al. ${ }^{15}$, o número de linfonodos analisados pelo patologista, após uma cirurgia oncológica, pode influenciar a sobrevida, independente do sítio do tumor. A teoria é baseada no fato de que 0 acometimento linfonodal determina de forma mais precisa o estadio da doença e, portanto, o prognóstico da mesma. Atualmente, além da importância do total de linfonodos dissecados, vem ganhando destaque o quociente linfonodos acometidos/ linfonodos dissecados ${ }^{16}$. Inicialmente, este parâmetro foi utilizado em trabalhos de câncer gástrico, em que se verificou que 0 quociente linfonodal era fator independente de sobrevida ${ }^{19,20}$.

No presente estudo, a relação entre prognóstico do câncer de cólon e o quociente de linfonodos acometidos/infonodos dissecados foi significante em relação à sobrevida apenas na análise univariada. $\mathrm{Na}$ análise multivariada, somente o estadiamento da doença permaneceu como uma variável independente para a sobrevida global, tanto na amostra global, quanto na amostra em que foram incluídos apenas os casos com no mínimo 10 linfonodos dissecados, apesar do pequeno número de pacientes prejudicar a interpretação. Possíveis explicações para o fato do quociente linfonodal não predizer a sobrevida de forma independente são: heterogeneidade da população estudada em relação ao estadiamento inicial do tumor e ao número de linfonodos dissecados no ato cirúrgico, curto tempo de seguimento dos pacientes e pequena casuística quando comparada a outros estudos. Vale ressaltar que também não foram avaliados outros fatores que poderiam ter impacto na sobrevida destes pacientes, como presença de comorbidades graves, complicações pós-operatórias, entre outros.

Ao contrário do nosso estudo, Berger et al. ${ }^{16}$, que foram os primeiros autores a utilizar o quociente linfonodal como fator prognóstico para câncer de cólon, observaram, na análise multivariada, que o quociente linfonodos acometidos/infonodos dissecados era um fator independente em relação não apenas à sobrevida global, mas também à sobrevida livre de doença, porém apenas nos pacientes com mais de 10 linfonodos dissecados. Meyers et al. ${ }^{18}$ avaliaram o quociente linfonodal em 1.648 pacientes com câncer retal, e também observaram que este quociente foi um fator preditivo de sobrevida global em cinco anos, independente do número total de linfonodos dissecados. É provável que estes estudos conseguiram mostrar a relação entre quociente linfonodal e sobrevida em pacientes com câncer de cólon, porque avaliaram grandes populações de pacientes. Além disso, Meyers et al. ${ }^{18}$ também demonstraram que, comparado a outros parâmetros, como o estadio TNM utilizado pelo AJCC (American Joint Committee on Cancer), o quociente linfonodal é o mais significante fator de prognóstico no que diz respeito à predição de sobrevida. Estudos de sobrevida em pacientes com câncer de cólon concluíram que a ressecçãa de poucos linfonodos é um indicador prognóstico ruim. Segundo Chen et al. ${ }^{21}$, a ressecção mínima de 15 linfonodos prolongam a média geral de sobrevida em II meses para pacientes em estadio I, 54 meses para estadio II, e 21 meses para estadio III. Diversos autores demonstraram que o número de linfonodos dissecados tem forte relação com a sobrevida dos pacientes, independente do estadio tumoral ${ }^{4,11-13}$.

A literatura recomenda que um mínimo de 10 linfonodos seja removido durante a cirurgia curativa para câncer colorretal. No presente estudo, a mediana de linfonodos ressecados foi de II,5 por paciente. Este é um dado que reflete a qualidade da cirurgia oncológica no sistema de saúde público da região do $A B C$ paulista. Os hospitais que integram este estudo fazem parte da Faculdade de Medicina do $A B C$ e, portanto, são instituições acadêmicas. Os resultados deste estudo mostram que a condução cirúrgica dos pacientes com tumores colorretais no sistema público do $A B C$ paulista se encontra dentro dos padrões internacionais de qualidade.

\section{ConClusão}

Conclui-se que no grupo de pacientes avaliados o estadiamento é o principal fator de prognóstico para sobrevida global. Provavelmente, em nosso meio, face ao grande número de pacientes com dissecção linfonodal subótima, a avaliação do quociente linfonodal como fator de prognóstico fica prejudicada. Estudos posteriores, envolvendo um maior número de pacientes, adequadamente abordados do ponto de vista cirúrgico, com dissecção linfonodal ótima, serão necessários para avaliar o real valor desta variável em relação à sobrevida destes pacientes.

\section{Conflito de interesse: não há.}

\section{SUMMARY}

\section{Positive / TOtal dISSECTEd LYMPH NOdes Ratio as a PROGNOStIC FACTOR IN COLON CANCER}

BACKGROUND. To evaluate the prognostic value of the ratio between positive and total dissected lymph nodes in patients with colon cancer who underwent primary tumor surgical resection.

Methods. Retrospective chart review of consecutive patients with colon cancer treated at hospitals affiliated to the $A B C$ 
Foundation School of Medicine, Santo André. Demographic data were collected as well as information on colon cancer, treatment and clinical outcomes.

RESULTS. One hundred and six patients were included. Mean age was $62.82 \pm 11.6$ years and most were men (53.8\%). Median number of lymph nodes dissected per patient was 11.5 (3 - 45 lymph nodes) and $58.5 \%$ had more than 10 dissected lymph nodes. The median follow-up was $25.05 \pm 15.21$ months (2 - 64 months). In univariate analysis for overall survival, lymph node ratio $(p=0.044)$, tumor stage $(p=0.001)$ and tumor recurrence $(p=0.058)$ were considered significant. In multivariate analysis only tumor stage was significantly associated with overall survival $(p=0.001)$.

CONCLUSION. In this limited retrospective series, the ratio between positive and dissected lymph nodes was not independently associated with overall survival among patients with colon cancer, when considered together with the pathological stage. Larger and prospective studies are warranted to define the impact of such ratio on the overall survival of colon cancer patients. [Rev Assoc Med Bras 2007; 53(6): 539-42]

KEY WORDS: Colonic neoplasms. Lymph nodes. Prognosis.

\section{REFERÊNCIAS}

I. Surveillance epidemiology and end results (SEER). National Cancer Institute. [cited 2007 may 22]. Avaliable from: http://seer.cancer.gov.

2. Estimativas da incidência de câncer no Brasil 2006. Ministério da Saúde: Instituto Nacional do Câncer. [citado 2007 maio 22]. Disponível em:http://www.inca.gov.br.

3. Tepper JE, O'Connell MJ, Niedzwiecki D. Impact of number of nodes retrieved on outcome in patients with rectal cancer. J Clin Oncol. 2001; 19:157-63.

4. LeVoyer TE, Sigurdson ER, Hanlon AR, Mayer RJ, Macdonald JS, Catalano PJ, et al. Colon cancer survival is associated with increasing number of lymph nodes analyzed: a secondary survey of intergroup trial INT-0089. J Clin Oncol. 2003;21:2912-9.

5. Dukes CE. The classification of cancer of the rectum. J Pathol Bacteriol. 1932;35:323-32.

6. Simpson WC, Mayo CW. The mural penetration of the carcinoma cell in the colon: anatomic and clinical study. Surg Gynecol Obstet. 1939;68:872-877.
7. Collaborative staging and coding manual. American Joint Committee on Cancer. [cited 2007 may 22]. Avaliable from: http:// www. cancerstaging.org.

8. Chau I, Cunningham D: Adjuvant therapy in colon cancer - what, when and how? Ann Oncol. 2006; 17:1347-59.

9. Benson $A B 3^{\text {rd }}$, Schrag D. Oncology recommendations on adjuvant chemotherapy for stage II cancer. J Clin Oncol. 2004:22:3408-19.

I 0. Compton CC, Fielding LP, Brugart LJ. Prognostic factors in colorectal cancer: College of American Pathologists Consensus Statement 1999. Arch Pathol Lab Med. 2000;124:979-94.

I I. Fielding LP, Arsenault PA, Chapuis PH. Working report to the World Congresses of Gastroenterology, Sydney 1990. J Gastroenterol Hepatol. 1991;6:325-44.

12. Goldstein NS, Weldon S, Coffey M. Lymph node recovery from colorectal resection specimens removed for adenocarcinoma: trends over time and a recommendation for a minimum number of lymph nodes to be removed. Am J Clin Pathol. 1996;106:209-16.

13. Wong JH, Severino R, Honnebier MB. Number of nodes examined and staging accuracy in colorectal carcinoma. J Clin Oncol. 1999;17:2896900.

14. Swanson RS, Compton CC, Stewart AK. The prognosis of T3NO colon cancer is dependent on the number of lymph nodes examined. Ann Surg Oncol. 2002; | 0:65-7|.

I 5. Sigurdson ER. Lymph node dissection: is it diagnostic or therapeutic? J Clin Oncol. 2003;21:965-7.

16. Berger AC, Sigurdson ER, LeVoyer T, Hanlon A, Mayer RJ, Mac Donald JS, et al. Colon cancer survival is associated with decreasing ratio of metastatic to examined lymph nodes. J Clin Oncol. 2005;23:8706-I2.

17. Costa LJM, Soares HP, Gaspar HA, Trujillo LG, Santos PX, Pereira R, et al. Ratio between positive lymph nodes and total dissected axillaries lymph nodes as na independent prognostic factor for disease-free survival in patients with breast câncer. Am J Clin Oncol. 2004;27:3046.

18. Meyers MO, Hollis DR, Mayer RJ. Ratio of metastatic to examined lymph nodes is a powerful predictor of overall survival in rectal cancer: an analysis of Intergroup 0I I4. In: 43rd American Society of Clinical Oncology Annual Meeting; June I-5, 2007. Chicago; 2007. Abstracts. n. 4006.

19. Bando E, Yonemura Y, Taniguchi K. Outcome of ratio of lymph node metastasis in gastric carcinoma. Ann Surg Oncol. 2002;9:775-84.

20. Inoue $K$, Nakane $Y$, liyama $H$. The superiority of ratio-based lymph node staging in gastric carcinoma. Ann Surg Oncol. 2002;9:27-34.

21. Chen SL, Bilchik AJ. More extensive nodal dissection improves survival for stages I to III of colon cancer. A population-based study. Ann Surg. 2006;244: 602-10.

Artigo recebido: 30/07/07

Aceito para publicação: $31 / 05 / 07$ 